\title{
Randomized, Controlled Evaluation of a Prototype Informed Consent Process for HIV Vaccine Efficacy Trials
}

\author{
*Anne S. Coletti, †Patrick Heagerty, \$Amy R. Sheon, §Michael Gross, "Beryl A. Koblin, \\ IIDavid S. Metzger, and \#George R. Seage III for the HIVNET VPS Protocol Team
}

*Family Health International, Research Triangle Park, North Carolina; †Fred Hutchinson Cancer Research Center, Seattle, Washington; + University of Michigan, Ann Arbor, Michigan; \$National Institute of Allergy and Infectious Diseases, Washington, D.C.; "New York Blood Center, New York, New York; IIUniversity of Pennsylvania, Philadelphia, Pennsylvania; and \#Harvard School of Public Health, Boston, Massachusetts

\begin{abstract}
Summary: Procedures must be developed to ensure that valid informed consent is obtained from participants in HIV vaccine efficacy trials. A prototype informed consent process was evaluated among 4,892 persons at high risk for HIV infection in the HIV Network for Prevention Trials Vaccine Preparedness Study (VPS), a prospective cohort study of HIV seroincidence in eight U.S. metropolitan areas. Twenty percent of VPS participants were selected at random to undergo the prototype informed consent process at VPS month 3. Participants' knowledge of 10 key HIV vaccine trial concepts and willingness to participate in HIV vaccine efficacy trials were assessed and compared at baseline and semiannually thereafter for 18 months. Knowledge of HIV vaccine trial concepts was low at baseline. Participation in the prototype process was associated with substantial and sustained increases in knowledge (relative risks for the 10 items, 1.04-2.26), which were of similar magnitude across HIV risk groups, race/ethnicity, and educational levels. It is recommended that the prototype informed consent process be adopted for future HIV vaccine efficacy trials as well as for clinical trials in other research areas. Key Words: Informed consent-HIV-HIV vaccinesRandomized trial—High-risk populations.
\end{abstract}

A substantial body of evidence suggests that the process of obtaining informed consent from participants in clinical research often is inadequate. Numerous reports have described research participants who neither were

This work was supported by the HIVNET, which is sponsored by the National Institute of Allergy and Infectious Diseases, National Institutes of Health, U.S. Department of Health and Human Services, in part through contract N01-AI-35176 with Abt Associates, Inc., contract N01-AI-45200 with the Fred Hutchinson Cancer Research Center, and subcontracts from Abt Associates, Inc., to the Denver Department of Public Health, Fenway Community Health Center, Howard Brown Health Center, New York Blood Center, New York University Medical Center, San Francisco Department of Public Health, University of Pennsylvania, and University of Washington.

The content of this publication does not necessarily reflect the views or policies of the U.S. Department of Health and Human Services, and mention of trade names, commercial products, or organizations does not imply endorsement by the U.S. Government.

Address correspondence and reprint requests to Dr. George R. Seage III, Harvard School of Public Health, Department of Epidemiology, 677 Huntington Avenue, Boston, MA 02115, U.S.A.; e-mail: gseage@ hsph.Harvard.edu

Manuscript received month May 16, 2001; accepted October 10, 2002. informed of nor consented to research participation (1-5) as well as participants who did not fully understand the nature and potential implications of research to which they consented (6-10). Such reports have prompted calls for improvement of the informed consent process based on available information as well as for additional research to guide further improvement (11-14).

Most studies of the informed consent process have focused on clinical trials of biomedical interventions and the potential risks of investigational products and required study procedures $(2,7)$. Potential efficacy trials of preventive HIV vaccines present unique additional challenges for protection of human subjects. In the United States, such trials will be undertaken among persons at high risk for HIV infection (15-17). Due to their sexual behaviors, drug use, illegal activities, race, educational attainment, economic status, and other factors, such persons are vulnerable to social harms-nonmedical adverse consequences- that may result from participation in HIV vaccine efficacy trials. Potential social harms 
include stigmatization/discrimination due to incorrect interpretation of results of standard HIV antibody tests, an incorrect presumption that participants in HIV vaccine efficacy trials are infected with HIV, and/or a conclusion that HIV vaccine trial participants belong to one or more stigmatized groups at "high risk" for HIV infection; HIV vaccine trial participants also may increase their HIV risk behaviors due to a belief in protection afforded by candidate HIV vaccines (18-24). Given these unique additional challenges, the U.S. Office for Technology Assessment (18) and others (20) have proposed guidelines for obtaining informed consent in future HIV vaccine efficacy trials. Other investigators have called for the development and evaluation of methods to convey this information and to document potential subjects' understanding of it, prior to implementation of large-scale trials $(22,25-29)$.

To develop methods to assure that participants in future HIV vaccine efficacy trials understand the implications and potential risks of participating, the HIV Network for Prevention Trials (HIVNET) developed a prototype informed consent process for a hypothetical future HIV vaccine efficacy trial. The prototype process was based on empirical research demonstrating that participant understanding of scientific information, research methods, and human subjects rights can be enhanced through the following: full, standardized disclosure of study-related information in both written and verbal formats (30); minimizing the reading level and enhancing the visual display of written documents $(9,31-33)$; requiring that nonphysician, noninvestigator "educators" describe and discuss the research with potential participants $(8,34)$; and providing adequate time and opportunity for participants to read, reflect upon, ask questions about, and discuss the research (32,35-37). This report presents the results of a field test of this prototype informed consent process conducted among 4,892 participants in the HIVNET Vaccine Preparedness Study (VPS).

\section{METHODS}

\section{Screening and Enrollment in the HIVNET VPS}

Between April and November 1995, men and women at increased risk for HIV infection who were from eight U.S. metropolitan areas (Boston, Chicago, Denver, New York, Philadelphia, Providence, San Francisco, and Seattle) were enrolled in the VPS, a prospective study of HIV seroincidence, HIV risk behaviors, and attitudes toward potential clinical trials of HIV prevention interventions, including vaccines. Study sites were located in clinic- and community-based settings including community health centers, hospital outpatient clinics, STD clinics, and store-front HIV counseling and research offices. Institutional review boards reviewed and approved the VPS in each location. Men who have sex with men, women at heterosexual risk, and male and female injection drug users were eligible for the study and were identified from existing studies $(n=1,974)$ and through referrals from enrolled study participants ( $n=1,043)$, outreach by study site staff $(n=977)$, media promotions such as print ads, posters, flyers, and hand cards distributed in community locations $(n=696)$, and other methods $(n=202)$.

After learning about the VPS, its requirements, risks, and benefits and providing written informed consent, study participants provided demographic and HIV risk behavior information via a standardized interview, received HIV pretest and risk reduction counseling, and provided serum samples for HIV antibody testing. Participants also completed a self-administered questionnaire on their baseline knowledge of HIV vaccine trials. After completing the questionnaire, participants read a brief fact sheet about HIV vaccine trials, discussed the fact sheet and any deficiencies in knowledge identified by the baseline questionnaire with a trained staff member, and then completed a selfadministered assessment of their willingness to participate in HIV vaccine trials. All data collection instruments were pilot-tested and refined based on the pilot test results prior to use.

Two weeks later, participants received their HIV test results and posttest counseling, and if HIV-seronegative, they were enrolled in the study. Thereafter, participants completed three semiannual study visits in which follow-up interviews and questionnaires were completed. As at baseline, HIV vaccine trial fact sheets were distributed and discussed at each follow-up visit; deficiencies in participant knowledge identified by follow-up questionnaires were discussed to improve participant understanding of misunderstood concepts at each time point. Consistent with local standards of practice, study participants at most sites were compensated for their time and effort in completing study visits. Forms of compensation varied across sites and included cash, gift certificates, merchandise, tokens for local transportation, and reimbursement for child care costs.

\section{Prototype Informed Consent Process}

In addition to their semiannual visits, 3 months after enrollment in the VPS, $20 \%$ of participants (hereafter referred to as "intervention participants") were selected at random to undergo a prototype informed consent process for potential future HIV vaccine efficacy trials. The selection algorithm was stratified by site and selected two participants at random from blocks of 10 participants. (Those not selected to undergo the prototype process hereafter are referred to as "control participants.") The HIVNET Statistical and Clinical Coordinating Center performed the selection and provided lists of selected participants to each study site.

After study staff carefully explained to intervention participants that they were not being asked to join an actual HIV vaccine efficacy trial but rather to assist in evaluating procedures for informed consent, the participants read an informational booklet that described a hypothetical HIV vaccine efficacy trial. Low-literacy participants were offered a verbatim audiotape recording of the form. The two-color 35-page booklet contained a series of numbered informational statements and supplemental text that explained the scientific and operational aspects of the trial. It also conveyed information required by federal regulations and was responsive to recommendations of the U.S. Office for Technology Assessment and others. (A sample excerpt from the booklet is included as Appendix A; copies of the booklet are available from the corresponding author.) The booklet was written at the eighth grade reading level (38).

Immediately after reading/listening to the booklet/tape, participants discussed any questions they had with a trained study staff member. 
Participants were encouraged to take the booklet/tape home with them to read/listen to it again and discuss it with others. They then were scheduled for a second visit 1 to 2 weeks later. At the second visit, participants discussed the material with a trained study staff member who probed participant understanding of key concepts using a semistructured script (see discussion guide provided to staff members for this purpose in Appendix B). Participants then completed a selfadministered questionnaire to assess their understanding of the hypothetical trial and their willingness to participate in it. This assessment included repetition of 10 knowledge items and one willingness item that participants had completed at baseline and would complete subsequently at follow-up. As at the baseline and semiannual follow-up visits, participants discussed any deficiencies in knowledge identified by the knowledge assessment with a trained staff member.

\section{Assessment Questionnaires and Statistical Analysis}

The knowledge questionnaire consisted of true-false style statements to which participants were asked to agree or disagree (Table 1). Participants also could indicate that they were "unsure" whether to agree or disagree. For analysis, all responses were scored as correct or incorrect; unsure responses were grouped with incorrect responses. Using an intent-to-treat approach, for each assessment time point, the mean number of correct responses to each knowledge item was computed for intervention and control participants. Intervention-control differences at each follow-up time point were evaluated using $t$ tests. The study sample size of 4,892 participants, with $20 \%$ undergoing the prototype informed consent process, provided $>99 \%$ power to detect a difference of one additional correct response among intervention participants, assuming a mean of 4.79 correct responses in the control group. Linear regression for longitudinal data (39) was used with data from all time points to evaluate whether intervention-control differences were modi- fied by three factors of a priori interest: educational level, race, and HIV risk group.

To elucidate the impact of the prototype informed consent process on each of the 10 key concepts, the percentage of intervention and control participants with correct responses was computed for each knowledge item at each time point. Relative risks (RRs) were computed to estimate the impact of participating in the prototype informed consent process on the probability of a correct response among intervention participants compared with control participants. In addition, conditional RRs indicating the likelihood of intervention participants responding correctly to each item, given a correct or incorrect response at the previous time point, were computed to assess the persistence of correct responses and correction of incorrect responses, respectively.

The willingness questionnaire asked participants to rate their willingness to participate in the hypothetical trial as definitely willing, probably willing, probably not willing, or definitely not willing. The percentage of intervention and control participants reporting each level of willingness and RRs estimating the impact of the prototype informed consent process on willingness were computed. When computing the RRs, definitely and probably willing participants were combined, as were definitely and probably unwilling participants.

\section{RESULTS}

\section{Study Population}

The demographic and HIV risk characteristics of the intervention and control participants were indistinguishable, as were follow-up visit completion rates (Table 2). There was no differential loss to follow-up across the

TABLE 1. Percent of intervention and control participants with correct responses

\begin{tabular}{|c|c|c|c|c|c|c|c|c|}
\hline & \multicolumn{2}{|c|}{ Baseline } & \multicolumn{2}{|c|}{ Month 6} & \multicolumn{2}{|c|}{ Month 12} & \multicolumn{2}{|c|}{ Month 18} \\
\hline & I & $\mathrm{C}$ & I & $\mathrm{C}$ & I & $\mathrm{C}$ & I & $\mathrm{C}$ \\
\hline $\begin{array}{l}\text { Q1: Some participants will get the real vaccine, and } \\
\text { some will get a placebo (an inactive substance). (T) }\end{array}$ & 65 & 65 & 93 & 81 & 94 & 86 & 96 & 91 \\
\hline $\begin{array}{l}\text { Q2: The study nurse will decide who gets the real } \\
\text { vaccine and who gets the placebo. }(F)\end{array}$ & 58 & 61 & 80 & 62 & 81 & 69 & 85 & 75 \\
\hline $\begin{array}{l}\text { Q3: People in the study are guaranteed a chance to be } \\
\text { in any future vaccine studies. (F) }\end{array}$ & 60 & 59 & 60 & 43 & 63 & 55 & 74 & 66 \\
\hline $\begin{array}{l}\text { Q4: Once a large-scale HIV vaccine study begins, we } \\
\text { can be sure the vaccine is completely safe. }(\mathrm{F})\end{array}$ & 53 & 53 & 63 & 56 & 64 & 57 & 64 & 61 \\
\hline $\begin{array}{l}\text { Q5: People in a vaccine study will know whether or } \\
\text { not they got the placebo because only the vaccine } \\
\text { will cause side effects. (F) }\end{array}$ & 51 & 53 & 84 & 73 & 87 & 79 & 88 & 84 \\
\hline $\begin{array}{l}\text { Q6: People in these studies will receive health care } \\
\text { for any medical problems they have, whether or not } \\
\text { the problems are related to the study. }(\mathrm{F})\end{array}$ & 50 & 52 & 72 & 55 & 78 & 65 & 84 & 74 \\
\hline $\begin{array}{l}\text { Q7: If people test HIV antibody-positive after getting } \\
\text { the vaccine, they may really be infected with HIV, } \\
\text { or they may just be responding to the vaccine. (T) }\end{array}$ & 46 & 46 & 80 & 66 & 83 & 74 & 86 & 80 \\
\hline $\begin{array}{l}\text { Q8: The vaccine may affect a participant's HIV } \\
\text { antibody test results. (T) }\end{array}$ & 40 & 42 & 81 & 62 & 84 & 76 & 88 & 82 \\
\hline $\begin{array}{l}\text { Q9: Only vaccines that are known to be at least } 50 \% \\
\text { effective at preventing HIV will be tested. (F) }\end{array}$ & 28 & 30 & 38 & 29 & 37 & 33 & 45 & 42 \\
\hline $\begin{array}{l}\text { Q10: An HIV vaccine could weaken the immune } \\
\text { system's ability to fight off HIV infection. (T) }\end{array}$ & 16 & 17 & 50 & 22 & 45 & 26 & 46 & 31 \\
\hline
\end{tabular}

I, intervention; C, control. Correct responses are shown in parentheses; T, true; F, false. 
TABLE 2. Demographics, HIV risk characteristics, and follow-up of intervention and control participants

\begin{tabular}{|c|c|c|c|c|}
\hline & \multicolumn{2}{|c|}{ Intervention } & \multicolumn{2}{|c|}{ Control } \\
\hline & $n$ & $\%$ & $n$ & $\%$ \\
\hline Overall & 984 & 100 & 3908 & 100 \\
\hline \multicolumn{5}{|l|}{ Sex } \\
\hline Male & 803 & 82 & 3224 & 83 \\
\hline Female & 181 & 18 & 684 & 17 \\
\hline \multicolumn{5}{|l|}{ Age, years } \\
\hline$<25$ & 111 & 11 & 413 & 11 \\
\hline $25-34$ & 427 & 43 & 1644 & 42 \\
\hline $35-44$ & 318 & 32 & 1292 & 33 \\
\hline $45+$ & 128 & 13 & 559 & 14 \\
\hline \multicolumn{5}{|l|}{ Race/ethnicity } \\
\hline White, non-Hispanic & 592 & 60 & 2381 & 61 \\
\hline Black, non-Hispanic & 212 & 22 & 814 & 21 \\
\hline Hispanic & 142 & 14 & 528 & 14 \\
\hline Asian/Pacific Islander & 17 & 2 & 85 & 2 \\
\hline Native American & 4 & $<1$ & 32 & 1 \\
\hline Other & 17 & 2 & 66 & 2 \\
\hline \multicolumn{5}{|l|}{ Educational level } \\
\hline Less than high school & 144 & 15 & 566 & 14 \\
\hline High school degree & 177 & 18 & 723 & 19 \\
\hline Some college & 243 & 25 & 1038 & 27 \\
\hline College degree & 239 & 24 & 870 & 22 \\
\hline Graduate school/degree & 181 & 18 & 711 & 18 \\
\hline \multicolumn{5}{|l|}{ Employment status } \\
\hline Employed full-time & 501 & 51 & 1974 & 51 \\
\hline Employed part-time & 127 & 13 & 433 & 11 \\
\hline Unemployed & 328 & 33 & 1369 & 35 \\
\hline Other & 28 & 3 & 131 & 3 \\
\hline \multicolumn{5}{|l|}{ HIV risk category } \\
\hline MSM & 658 & 67 & 2599 & 67 \\
\hline MIDU & 145 & 15 & 625 & 16 \\
\hline WSM & 113 & 11 & 398 & 10 \\
\hline WIDU/WSM & 68 & 7 & 286 & 7 \\
\hline \multicolumn{5}{|l|}{ Follow-up visit completion } \\
\hline Month 6 & 912 & 93 & 3660 & 94 \\
\hline Month 12 & 865 & 88 & 3501 & 90 \\
\hline Month 18 & 824 & 84 & 3352 & 86 \\
\hline
\end{tabular}

MSM, men who have sex with men; MIDU, male injection drug users; WSM, women at heterosexual risk; WIDU/WSM, female injection drug users with heterosexual risk.

intervention and control groups with respect to participant race, educational level, or HIV risk group.

\section{Delivery of the Prototype Informed Consent Process}

Of the 984 VPS participants selected at random to complete the prototype informed consent process, 774 (79\%) completed all of the prototype informed consent procedures (Fig. 1). An additional 46 intervention participants completed only the initial prototype visit. Selected participants who did not complete the prototype process were younger (64\% vs. $52 \%$, respectively, were younger than 35 years of age) and of lower educational attainment (68\% vs. 55\%, respectively, held less than a college degree) than selected participants who completed the process; their characteristics were otherwise compa- rable with those shown in Table 2. Due to errors at the study sites, six control participants completed the prototype informed consent process.

Among intervention participants who completed at least the initial prototype visit, educational discussions took a median of 14 minutes to complete (interquartile range, 9-21 minutes). The second prototype visit took place a median of 8 days after the initial visit (interquartile range, 7-14 days). Eighty percent of participants who completed the second visit reported having reread the booklet or listened to the tape again at least once between visits. Seventy-four percent discussed the information with at least one other person; among these participants, $60 \%$ discussed the information with friends, $45 \%$, with a spouse or primary partner, $14 \%$, with a co-worker, $12 \%$, with an immediate family member, and $10 \%$, with another person. Educational discussions at the second prototype visit took a median 15 minutes to complete (interquartile range, 9-24 minutes).

\section{Knowledge of HIV Vaccine Trial Concepts}

Knowledge levels among intervention and control participants in the two groups were comparable at baseline, with both groups responding correctly to fewer than one half of the items (4.68 correct among intervention participants and 4.79 correct among control participants). Both groups improved in knowledge over time, as expected given the standard semiannual VPS proce-

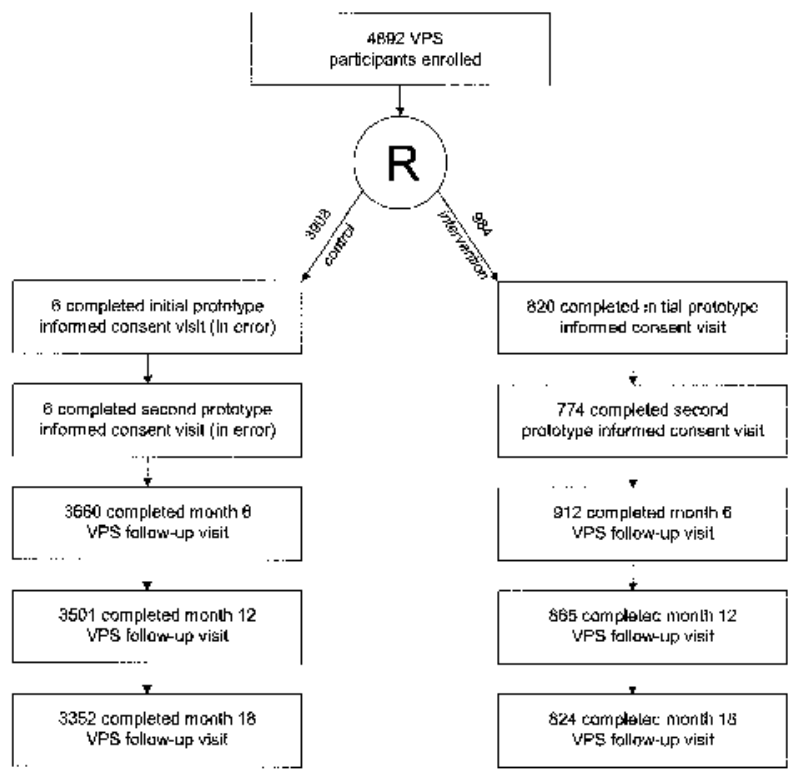

FIG. 1. Randomization scheme and follow-up among intervention and control participants. VPS, Vaccine Preparedness Study; $\mathrm{R}$, randomization. 
dures described above. However, intervention participants responded correctly to significantly more items than did control participants at each follow-up time point (7.00 vs. 5.49 at month $6,7.17$ vs. 6.19 at month 12 , and 7.57 vs. 6.86 at month 18 , respectively; $p<.05$ at all time points). Linear regression for longitudinal data indicated that the difference between intervention and control participants was not modified by participant race (Wald $\chi^{2}=6.4 ; p=.27$ ), educational level (Wald $\chi^{2}=5.1$; $p=.28$ ], or HIV risk group (Wald $\chi^{2}=1.3 ; p=.72$ ).

The percentage of intervention and control participants responding correctly to each knowledge item at baseline and study months 6,12 , and 18 is presented in Table 1. Baseline percentages were comparable for all items (with only six items responded to correctly by at least one half of the participants in each group), and both groups had increased percentages over time for each item. Although the magnitude of improvement varied across items, the percentage of intervention participants who responded correctly to each item at each follow-up time point was higher than that of control participants.

At month 6, when the most pronounced effects of the intervention were expected, RRs estimating the effect of the intervention on the likelihood of responding correctly to any item ranged from 1.14 to 2.26 , and all were significantly greater than the null (Fig. 2). Significant differences persisted between the intervention group and the control group for all items at month 12 (with RRs from 1.09 to 1.74) and for all but two items at month 18 (with RRs from 1.04 to 1.48). The magnitude of the RRs for each item decreased over time, due principally to increases in knowledge among control participants rather than to decays in knowledge among intervention participants.
Intervention and control participants' RRs of retaining correct responses and correcting incorrect responses are compared in Table 3. For all but one item, intervention participants were significantly more likely than control participants to retain correct baseline responses at month 6 (with RRs ranging from 1.05 to 1.32). Results were similar at months 12 and 18 , although differences between the two groups decreased somewhat for most items, due to improvements among control participants over time rather than to decays in knowledge among intervention participants.

Even larger effects were observed in terms of intervention participants correcting incorrect baseline responses at month 6; RRs ranged from 1.32 to 2.81, and all were significantly greater than the null. Little to no effect in this regard was observed at months 12 and 18, indicating that the impact of the prototype informed consent process on improving understanding was concentrated immediately following the intervention.

\section{Willingness to Participate in HIV Vaccine Trials}

Intervention and control participants reported similar levels of willingness to participate in an HIV vaccine efficacy trial at baseline, with approximately threequarters of participants in both groups reporting either definite or probable willingness to participate. At month $6,67 \%$ of intervention participants reported being either probably or definitely willing, compared with $71 \%$ of control participants $(\mathrm{RR}=0.94 ; 95 \% \mathrm{CI}, 0.89-0.99)$; results were similar at months $12(65 \%$ vs. $70 \%$, respectively, probably or definitely willing; $\mathrm{RR}=0.94 ; 95 \%$ CI, $0.89-0.99)$ and 18 (62\% vs. 66\%, respectively,

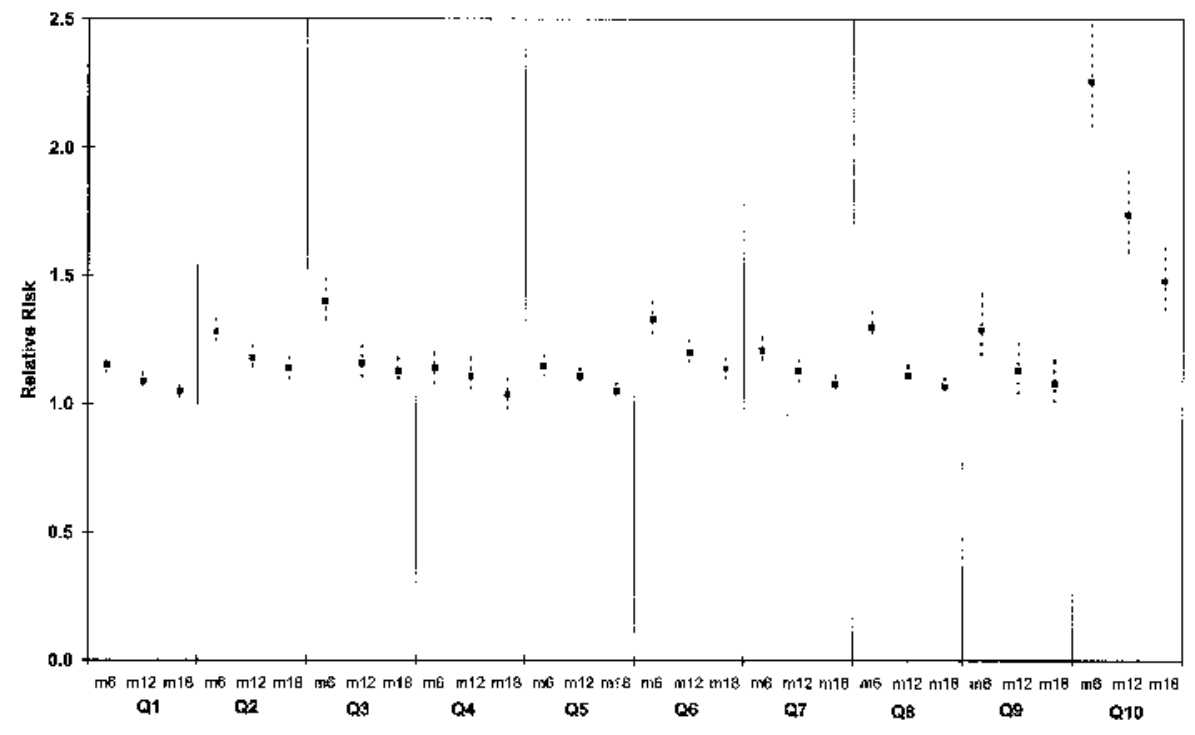

FIG 2. Relative risks and $95 \%$ confidence intervals comparing correct responses among intervention and control participants. 
TABLE 3. Relative risks comparing correct response among intervention and control participants, conditioned on response at prior assessment

\begin{tabular}{|c|c|c|c|c|c|c|}
\hline & \multicolumn{2}{|c|}{ Month 6} & \multicolumn{2}{|c|}{ Month 12} & \multicolumn{2}{|c|}{ Month 18} \\
\hline & $\begin{array}{l}\text { Correct at } \\
\text { baseline }\end{array}$ & $\begin{array}{l}\text { Incorrect at } \\
\text { baseline }\end{array}$ & $\begin{array}{l}\text { Correct at } \\
\text { month } 6\end{array}$ & $\begin{array}{l}\text { Incorrect at } \\
\text { month } 6\end{array}$ & $\begin{array}{l}\text { Correct at } \\
\text { month } 12\end{array}$ & $\begin{array}{l}\text { Incorrect at } \\
\text { month } 12\end{array}$ \\
\hline $\begin{array}{l}\text { Q1: Some participants will get the real vaccine, and } \\
\text { some will get a placebo (an inactive substance). (T) }\end{array}$ & $1.05^{a}$ & $1.43^{a}$ & 1.02 & 1.15 & $1.01^{a}$ & 1.09 \\
\hline $\begin{array}{l}\text { Q2: The study nurse will decide who gets the real } \\
\text { vaccine and who gets the placebo. }(F)\end{array}$ & $1.17^{a}$ & $1.63^{a}$ & $1.08^{a}$ & 0.98 & $1.07^{a}$ & 1.03 \\
\hline $\begin{array}{l}\text { Q3: People in the study are guaranteed a chance to be } \\
\text { in any future vaccine studies. }(F)\end{array}$ & $1.32^{a}$ & $1.64^{a}$ & $1.08^{a}$ & 0.88 & $1.06^{a}$ & 1.07 \\
\hline $\begin{array}{l}\text { Q4: Once a large-scale HIV vaccine study begins, we } \\
\text { can be sure the vaccine is completely safe. }(\mathrm{F})\end{array}$ & $1.08^{a}$ & $1.25^{a}$ & $1.07^{a}$ & 1.03 & 0.99 & 1.02 \\
\hline $\begin{array}{l}\text { Q5: People in a vaccine study will know whether or } \\
\text { not they got the placebo because only the vaccine } \\
\text { will cause side effects. (F) }\end{array}$ & $1.05^{a}$ & $1.33^{a}$ & $1.07^{a}$ & 1.00 & 1.02 & 0.98 \\
\hline $\begin{array}{l}\text { Q6: People in these studies will receive health care } \\
\text { for any medical problems they have, whether or not } \\
\text { the problems are related to the study. }(\mathrm{F})\end{array}$ & $1.20^{a}$ & $1.63^{a}$ & $1.11^{a}$ & 0.97 & $1.03^{a}$ & $1.28^{a}$ \\
\hline $\begin{array}{l}\text { Q7: If people test HIV antibody-positive after getting } \\
\text { the vaccine, they may really be infected with HIV, } \\
\text { or they may just be responding to the vaccine. (T) }\end{array}$ & $1.11^{a}$ & $1.32^{a}$ & $1.04^{a}$ & 1.14 & $1.04^{a}$ & 1.03 \\
\hline $\begin{array}{l}\text { Q8: The vaccine may affect a participant's HIV } \\
\text { antibody test results. (T) }\end{array}$ & $1.17^{a}$ & $1.46^{a}$ & $1.03^{a}$ & $0.82^{a}$ & 1.01 & 0.98 \\
\hline $\begin{array}{l}\text { Q9: Only vaccines that are known to be at least } 50 \% \\
\text { effective at preventing HIV will be tested. }(\mathrm{F})\end{array}$ & 1.03 & $1.56^{a}$ & 1.13 & 0.97 & $1.13^{a}$ & 0.96 \\
\hline $\begin{array}{l}\text { Q10: An HIV vaccine could weaken the immune } \\
\text { system's ability to fight off HIV infection. (T) }\end{array}$ & $1.20^{a}$ & $2.81^{a}$ & $1.43^{a}$ & 1.08 & $1.36^{a}$ & 0.98 \\
\hline
\end{tabular}

${ }^{a} p<.05$. Correct responses are shown in parentheses; T, true; F, false.

probably or definitely willing; $\mathrm{RR}=0.95 ; 95 \% \mathrm{CI}$, $0.90-1.01)$.

\section{DISCUSSION}

The HIVNET VPS provided an important opportunity to evaluate prototype procedures for obtaining informed consent in future HIV vaccine efficacy trials prior to undertaking such trials. The evaluation indicated that the prototype procedures - which included intensive educational efforts at the outset, followed by targeted semiannual "booster" educational sessions-were associated with substantial, statistically significant, and sustained increases in participant knowledge of key concepts. The impact of the intervention was similar for all participants, regardless of race, educational level, and HIV risk group. This finding substantiates previous research that has indicated, for example, that injection drug users are equally capable of comprehending informed consent concepts as other participants in a phase 2 HIV vaccine trial (40). Thus, exclusion of any demographic or HIV risk group from future HIV vaccine efficacy trials-based on concerns related to ability to comprehend key HIV vaccine trial concepts-would be unwarranted. Prototype procedures also were associated with a negligible reduction in willingness to enroll in HIV vaccine efficacy trials. Therefore, no evidence was provided that effective informed consent procedures will adversely impact accrual into future HIV vaccine trials in the United States.

The magnitude of the increase in participant knowledge levels associated with the prototype informed consent process is worthy of additional comment. Whereas the relative improvement among the intervention participants compared with the control participants-as measured by the RRs in Figures 1 and 2-is fairly large, the absolute improvement in overall knowledge score among intervention participants appears more modest. It should be noted, however, that since each of the items included on the knowledge questionnaire represents a concept considered critical to a potential HIV vaccine trial participant's decision to enroll in a trial, an improvement of even one additional correct response is quite meaningful. In addition, our use of an intent-to-treat approach for this analysis results in conservative estimates of the impact of the prototype informed consent process, since $>20 \%$ of participants included in the intervention group did not in fact complete the prototype process. For example, an "as treated" analysis of the percentage of correct items compared across intervention and control groups would result in observed knowledge score differences of 1.87 , 1.24 , and 0.83 at study months 6,12 , and 18 , respectively (vs. differences of $1.52,0.98$, and 0.71 , respectively, in the intent-to-treat analysis). 
On the other hand, this evaluation identified some persistent knowledge deficits. At no time did 100\% of intervention participants respond correctly to any knowledge item, and fewer than three-quarters of intervention participants responded correctly to several items. Perhaps of greatest concern, participants' most common and most persistent knowledge deficits were related to the unknown safety and unproven efficacy of candidate HIV vaccines. This observation is consistent with previous experience suggesting that persons tend to overlook, misinterpret, and/or fail to recall potentially threatening information (41). It also is consistent with the so-called "therapeutic misconception," in which research participants suppress an understanding of research methodology in favor of a belief that investigators have designed their treatment in a clinical trial to meet their individual needs (8). In this case, VPS participants may have suppressed information conveyed to them during the prototype informed consent process in favor of a belief that an HIV vaccine efficacy trial would only be undertaken with a promising (i.e., safe and effective) candidate vaccine. Thus, for future HIV vaccine efficacy trials, it will be critical to emphasize and assure participants' understanding that candidate vaccines entered into such trials are still subject to continued safety evaluations and are of unknown efficacy.

The size of the VPS and the opportunity it provided to perform a prospective, randomized, controlled trial of the prototype informed consent procedures afforded this evaluation protection against common methodological pitfalls in epidemiologic research, particularly selection bias, confounding, and insufficient power to detect meaningful intervention effects. Although the results of this evaluation may not be broadly generalizable, they are likely relevant to other U.S. populations at high risk for HIV infection who may be recruited to participate in future HIV vaccine efficacy trials, and they clearly are generalizable to populations recruited from the VPS for other clinical research. As such, they were used to formulate informed consent procedures for a phase $2 \mathrm{HIV}$ vaccine trial that recruited high-risk participants from the VPS (42).

For that phase 2 trial, in consultation with study site staff, community educators, and community advisory board members, the VPS informational booklet was revised to further clarify concepts that were most commonly misunderstood. In addition, consistent with recommendations that video presentations be included in the informed consent process (43), a videotape was prepared to serve as a standardized introduction to the informed consent process for each potential trial participant. The VPS knowledge assessment and debriefing procedures were refined to ensure that only persons who could demonstrate understanding of at least 14 of $15 \mathrm{key}$ trial concepts in no more than three attempts to complete the assessment instrument were permitted to enroll in the trial. As applied in this trial, the informed consent process was a success, in that no potential participants were excluded from the trial due to inability to meet the demonstrated knowledge requirements. After enrollment, educational "boosters" were provided at scheduled followup visits to ensure continued understanding and ongoing informed consent throughout the duration of the study.

In conclusion, this evaluation provided evidence that U.S. populations targeted for participation in HIV vaccine efficacy trials-even those of low educational attainment - can be informed about such trials and, perhaps equally important, that knowledge gained through prototype educational procedures can be retained over time with minimal reinforcement. This evaluation also identified subject areas requiring additional attention as part of actual informed consent procedures. Although the details of the process and associated materials will require study-specific tailoring, it is recommended that an objective assessment be used to assess participant understanding of key trial concepts, that the results of the assessment be used to guide additional educational efforts required to ensure participant understanding, and that potential participants who are unable to demonstrate adequate understanding of key concepts not be enrolled in clinical research. It also is recommended that elements of the prototype informed consent process, including the knowledge assessment, be implemented throughout the duration of a clinical trial, to ensure ongoing informed consent among enrolled participants as well as to provide quality assurance information on the delivery of the informed consent process over time.

Acknowledgments: The following institutions and persons participated in the HIVNET VPS Protocol Team: HIVNET Domestic Master Contractor, Abt Associates, Inc., M. Gross and G. Seage III; HIVNET Statistical and Clinical Coordinating Center, Fred Hutchinson Cancer Research Center, T. Fleming and S. Self; HIVNET Central Laboratory, Viral and Rickettsial Disease Laboratory, California Department of Health Services, M. Ascher and H. Sheppard; HIVNET Repository Contractor, Biomedical Research, Inc., J. Leef and R. Rowe; Denver Department of Public Health, F. Judson; Fenway Community Health Center, K. Mayer; Howard Brown Health Center, D. McKirnan and T. Warne; New York Blood Center, B. Koblin and C. Stevens; New York University Medical Center, M. Marmor; San Francisco Department of Public Health, S. Buchbinder; University of Pennsylvania, D. Metzger and G. Woody; University of Washington, C. Celum; National Institute of Allergy and Infectious Diseases, R. Hoff, D. Lawrence, M. McCauley, W. Rida, and A. Sheon; and Centers for Disease Control and Prevention, K. MacQueen. 
The authors acknowledge the contributions of the site coordinators, other site staff, and community advisory board members as well as the dedication of VPS participants.

\section{REFERENCES}

1. Beecher HK. Ethics and clinical research. N Engl J Med 1966;274: 1354-60.

2. Gray BH. Human subjects in medical experimentation. New York: Wiley, 1975.

3. Ad Hoc Advisory Panel. Final report on the Tuskeegee Syphilis Study. Washington, DC: US Government Printing Office, 1973:5-15.

4. Howard JM, DeMets D, the BHAT Research Group. How informed is informed consent? The BHAT experience. Control Clin Trials 1981;2:287-303.

5. Cancer Research Campaign Working Party in Breast Conservation. Informed consent: ethical, legal, and medical implications for doctors and patients who participate in randomised clinical trials. BMJ 1983;286:1117-21.

6. Schultz AL, Pardee GP, Ensinck JW. Are research subjects really informed? West J Med 1975;123:76-80.

7. Lynöe N, Sandlund M, Dahlqvist G, et al. Informed consent: study of the quality of information given to participants in a clinical trial. BMJ 1991;303:610-3.

8. Applebaum PS, Roth LH, Lidz CW, et al. False hopes and best data: consent to research and the therapeutic misconception. Hastings Cent Rep 1987;April:20-4.

9. Epstein LC, Lasagna L. Obtaining informed consent: form or substance. Arch Intern Med 1969;123:682-8.

10. Advisory Committee on Human Radiation Experiments. Final report. Washington, DC: US Government Printing Office, 1995.

11. Benson PR, Roth LH, Winslade WJ. Informed consent in psychiatric research: preliminary findings from an ongoing investigation. Soc Sci Med 1985;20:1331-41.

12. MacKillop WJ, Johnston P. Ethical problems in clinical research: the need for empirical research studies of the clinical trials process. J Chron Dis 1986;39:177-88.

13. Taub HA. Comprehension of informed consent for research: issues and directions for future studies. IRB 1986;November-December:7-10.

14. Stanley B, Sieber JE, Melton GB. Empirical studies of ethical issues in research: a research agenda. Am Psychol 1987;42:735-41.

15. Vermund SH, Fischer RD, Hoff R, et al. Preparing for HIV vaccine efficacy trials: partnerships and challenges. AIDS Res Hum Retroviruses 1993;10(suppl 1):127-32.

16. Hoff R, Barker L. Trial objectives and end points for measuring the efficacy of HIV vaccines. Infect Agents Dis 1995;4:95-101.

17. Rida W, Fast P, Hoff R, et al. Intermediate-size trials for the evaluation of HIV vaccine candidates: a workshop summary. J Acquir Immune Defic Syndr Hum Retrovirol 1997;16:195-203.

18. US Congress, Office of Technology Assessment. Adverse reactions to HIV vaccines: medical, ethical, and legal issues. OTABP-H-163. Washington, DC: US Government Printing Office, 1995.

19. Sheon AR. Preventing discrimination against volunteers in preventive HIV vaccine efficacy trials: conference summary. AIDS Hum Retrovir 1995;11:1309-11.

20. Grady C. The search for an AIDS vaccine: ethical issues in the development and testing of a preventive HIV vaccine. Bloomington, IN: Indiana University Press, 1995:34-140.

21. Collins C. Sustaining support for domestic HIV vaccine research: social issues over the long haul of human trials. Monograph series occasional paper no. 2. San Francisco, CA: Center for AIDS Prevention Studies, University of California San Francisco, 1996.

22. AIDS Action Foundation. HIV preventive vaccines: social, ethical, and political considerations for domestic efficacy trials. Washington, DC: 1994.

23. Sheon AR, Wagner L. McElrath MJ, et al. Preventing discrimination against volunteers in prophylactic HIV vaccine trials: lessons from the phase II trial. J Acquir Immune Defic Syndr Hum Retrovirol 1998;19:519-26.

24. Chesney MA, Chambers DB, Kahn JO. Risk behavior for HIV infection in preventive HIV vaccine trials: a cautionary note. $J$ Acquir Immune Defic Syndr 1997;16:266-71.

25. Hodel D. HIV preventive vaccine efficacy trials in the US: overview of target communities concerns. AIDS Res Hum Retroviruses 1994;10(supp1 2):S255-6.

26. Turner CF, Sheon AR. Behavioral studies relevant to vaccine trial preparation: an introduction. AIDS Res Hum Retroviruses 1994;10 (suppl 2):S273-6.

27. Temoshok LR. Behavioral research contributions to planning and conducting HIV vaccine efficacy studies. AIDS Res Hum Retroviruses 1994;10(suppl 2):S277-80.

28. Sugarman J. Toward achieving meaningful informed consent in AIDS vaccine trials with injection-drug users. AIDS Public Policy Journal 1994;9:167-72.

29. Lurie P, Bishaw M, Chesney M, et al. Ethical, behavioral, and social aspects of HIV vaccine trials in developing countries. JAMA 1994;271:295-301.

30. Simes RJ, Tattersall MHN, Coates AS, et al. Randomised comparison of procedures for obtaining informed consent in clinical trials of treatment for cancer. BMJ 1986;293:1065-8.

31. Tymchuk AJ, Ouslander JG, Rader N. Informing the elderly: a comparison of four methods. J Am Geriatr Soc 1986;34:818-22.

32. Young DR, Hooker DT, Freeberg FE. Informed consent documents: increasing comprehension by reducing reading level. IRB 1990;May-June:1-5.

33. Peterson BT, Clancy SJ, Champion K, et al. Improving readability of consent forms: what the computers don't tell you. IRB 1992;November-December:6-8.

34. Muss HB, White DR, Michielutte R, et al. Written informed consent in patients with breast cancer. Cancer 1979;43:1549-56.

35. Levine RJ. More on making consent forms readable [editorial]. IRB 1982;January-February:8-9.

36. Meisel A. More on making consent forms readable [editorial]. IRB 1982;January-February:9.

37. Morrow G, Gootnick J, Schmale A. A simple technique for increasing cancer patients' knowledge of informed consent to treatment. Cancer 1978;42:793-9.

38. Based on analysis by RightWriter [computer program]. Version 3.1. RightSoft, Inc., 1989.

39. Liang K-Y, Zeger SL. Longitudinal data analysis using generalized linear models. Biometrika 1986;73:13-22.

40. Harrison K, Vlahav D, Jones K, et al. Medical eligibility, comprehension of the consent process, and retention of injection drug users recruited for an HIV vaccine trial. JAIDS 1995;10:386-90.

41. Silva MC, Sorrell JM. Enhancing comprehension of information for informed consent: a review of empirical research. IRB 1988;January-February:1-5.

42. Belshe RB, Stevens C, Gorse GJ, et al. Safety and immunogenicity of a canarypox-vectored human immunodeficiency virus type 1 vaccine with or without gp120: a phase 2 study in higher- and lower-risk volunteers. J Infect Dis 2001;183:1343-52.

43. Barbour GL, Blumenkrantz MJ. Videotape aids informed consent decision. JAMA 1978;240:2741-2. 
APPENDIX A. Excerpt from prototype informational booklet

\section{People in the study will be chosen at random to receive either the vaccine or the placebo.}

You will be chosen to get either the vaccine or placebo "at random." This means it is like flipping a coin or throwing dice. Since half of the people in the study will get the vaccine and half will get the placebo, you have a 50-50 chance of getting the vaccine or the placebo.

15. You will not know until the end of the study whether you got the vaccine or the placebo.

NO ONE will be told whether they got the vaccine or placebo until the study is over. Even the doctors, nurses, and other staff here will NOT know whether they gave you the vaccine or the placebo. That means you may have to wait at least two years after your final injection to find out. Even though you will only be in the study for two years, other study participants may finish after you.

This could change only if you have a medical emergency that makes it necessary to know whether you received vaccine. This will be determined by an independent physician. If you find out whether you got the vaccine because of an emergency, you will still be in the study and come back for follow-up visits.

A group of outside experts will be watching the study to protect the safety of the participants. If during the study, these experts see any clear difference between the vaccine group and the placebo group, the study will be stopped and everyone will be told. [See also \#59 and \#60.]

16. People who join this study must continue to be careful to avoid HIV.

No one knows how well the study vaccine works, if at all. [See \#9.] We do know that the vaccine won't work for everyone who gets it.

Since you will not know whether you got the vaccine or placebo, you MUST continue to be careful to avoid behaviors that could give you HIV.

You will be told by staff here how to avoid HIV. They will remind you often how important it is to keep trying to protect against HIV. Also, you may schedule extra visits during the study to talk to the counselors here about HIV and how to avoid HIV infection. [See also \#27.]

Note: Cross-references in brackets refer to other sections of the booklet not reproduced here.

APPENDIX B. Prototype informed consent discussion guide for study site educators

\begin{tabular}{|c|c|}
\hline Discussion question: & Used to probe participant understanding of: \\
\hline $\begin{array}{l}\text { Are there things about being in a vaccine study } \\
\text { like this that you would be worried about? }\end{array}$ & $\begin{array}{l}\text { The study vaccine cannot cause HIV infection. } \\
\text { Although the vaccine has been safe in the short-term, it's long-term effects and its } \\
\text { effects in pregnancy are unknown: safety is still being studied. } \\
\text { - Study participation could result in social harm. } \\
\text { - Personal participant information, including the fact that they are in the study, will be } \\
\text { protected to the greatest extent possible. }\end{array}$ \\
\hline $\begin{array}{l}\text { How do you think it would affect your life to } \\
\text { be in a study like this? }\end{array}$ & $\begin{array}{l}\text { Study length and visit requirements. } \\
\text { - Services provided (and not provided) by the study. } \\
\text { Perceived risks and benefits of the study; potential for social harm. }\end{array}$ \\
\hline $\begin{array}{l}\text { Do you think that being in a preventive HIV } \\
\text { vaccine study would help you avoid getting } \\
\text { infected with HIV? Explain. }\end{array}$ & $\begin{array}{l}\text { - Unknown efficacy of study vaccine. } \\
\text { - Random, blind assignment to vaccine or placebo. } \\
\text { - Study participants will be counseled to practice safe behaviors throughout the study. }\end{array}$ \\
\hline $\begin{array}{l}\text { If you joined a study like this, how would you } \\
\text { be able to find out whether or not you had } \\
\text { become infected with HIV? }\end{array}$ & $\begin{array}{l}\text { The importance of getting tested at the study site. } \\
\text { The concept of periodic testing as a regular part of study participation. }\end{array}$ \\
\hline $\begin{array}{l}\text { If you wanted to tell a friend or family } \\
\text { member that you were in a study like this, } \\
\text { how would you describe it to them? }\end{array}$ & $\begin{array}{l}\text { Overall study design, duration, and procedural requirements. } \\
\text { This question also will get at the likelihood the participant would describe the study in } \\
\text { a way that causes others to mistakenly think he/she is HIV-infected. }\end{array}$ \\
\hline $\begin{array}{l}\text { What would you do if you joined the study } \\
\text { and then you didn't feel comfortable about } \\
\text { the way you were treated in the study? }\end{array}$ & $\begin{array}{l}\text { The role of the IRB and the Community Advisory Board. } \\
\text { Participants can leave the study at any time. } \\
\text { - Participants who leave the study can still keep coming back to the site for other } \\
\text { programs or services to which they are entitled. }\end{array}$ \\
\hline $\begin{array}{l}\text { When you read the booklet about the vaccine } \\
\text { study, do you remember any things about } \\
\text { the study procedures that were put in place } \\
\text { to help protect your privacy and safety? }\end{array}$ & $\begin{array}{l}\text { Interpretation of animal and Phase I/II safety data. } \\
\text { Confidentiality protections (ID codes, locked/restricted files, etc). } \\
\text { Medical follow-up provided as part of the study/medical care that is the responsibility } \\
\text { of the participant and his/her insurer. } \\
\text { The role of the DSMB. }\end{array}$ \\
\hline
\end{tabular}

